\title{
AN APPROACH TO WEB SERVICE SELECTION BASED ON COMPOSITE QOS PARAMETERS
}

\author{
Mehrnoosh Kheradmand', Homayun Motameni ${ }^{2}$ \\ 1 Student, Sari Branch, Islamic Azad University, Sari, Iran, e-mail: m.kheradmand.85@gmail.com \\ 2 Sari Branch, Islamic Azad University, Sari, Iran, e-mail: motameni@iausari.ac.ir
}

Received: 2015.01.26

Accepted: 2015.05.08

Published: 2015.06.01

\begin{abstract}
Quality of service (QoS) is an important attribute for selecting a service during the service composition process. Although availability and reliability have been considered as the predominant factors for estimating reputation, two aspects are missing in the literature. First, their use is limited to composite service level and does not count on the atomic level. Second, their combined effect is not evaluated. Better estimation of QoS can be done with both factors considered together, whereas when taken separately for computing reputation, availability will give the probability of a service being up/running and reliability will analyze the change in failure trend of that service. In this research, a mathematical modeling of predominant QoS factors, availability and reliability of atomic services using Markov Chain model and Weibull analysis respectively, are suggested. Also importance of modeling reputation as an aggregation of availability and reliability has been explained. This research concludes two results. First, counting on probability of a service being up/running and its failure trend, together, results in a better estimation of its behavior and helps selecting the most appropriate one. Second, this resulted in selection of a service with higher reputation but lower usage cost, as opposed to using a single factor that resulted in higher reputation with higher cost.
\end{abstract}

Keywords: web service, web service selection, quality of service, QoS composition, service reputation.

\section{INTRODUCTION}

Web services encapsulate operations and intelligence sources, and available them by using standard programming interfaces through the web. With the increasing number of web services that provide similar functions, how to find appropriate web services for user requirements becomes more and more important [5]. User requirements include functional and non-functional requirement. Functional requirements specify the behavioral characteristics and non-functional requirements specify the quantitative characteristics. The selection of an atomic service is based on various quality of service (QoS) parameters. Services are ranked based on their QoS values and selection is done based on rank. One of the QoS ranking crite- ria is reputation factor. Availability and reliability are considered as predominant factors for computing reputation that analyze the probability of a service being up/running and the change in failure trend of that service respectively. Better estimation of QoS can be done with both factors considered together, whereas when taken separately for computing reputation, may not generate appropriate result. Let us consider the following scenario: Let there be two services $S_{A}, S_{B}$ with $A_{A}=0.8612$, $A_{B}=0.8607, R_{A}=0.6664, R_{B}=0.7146$ as availability and reliability of $S_{A}, S_{B}$ respectively. Now, if reputation is computed only on availability then service $S_{A}$ would be the choice for selection and if reputation is computed only by comparing their reliability then service $S_{B}$ will be selected. So, the study of the behavior of a service in terms of its 
availability and reliability over a sample space would provide better knowledge about its suitability for selection. In this paper to quantify the predominant factors of QoS, availability and reliability of atomic services use Markov Chain model and Weibull analysis respectively. In continue in section 2 the related work and in section 3 the proposed approach is presented. In section 4 the simulation and in section 5 the result of experiments is explained. Finally, in section 6 the general conclusion and in section 7 future work is presented.

\section{RELATED WORK}

Ranking of services and computing QoS is done in two ways: Ranking of atomic services and ranking of composite services. Most researches have focused on ranking composite services and pay less than the atomic services.

In [1] an approach for ranking atomic services using reputation factor for computing QoS is used. This approach suggests a mathematical modeling of factors availability and reliability of atomic services that using Markov Chain model and Weibull analysis respectively. Also reputation is modeled as an aggregation of availability and reliability factors, this composition is done by multiplication operation.

Research in the field of Web service composition based on QoS can be broadly put into two directions. One focuses on how various QoS attributes can be quantified for their effective use and the other focuses on their use at various stages of service selection or composition [1].

In the direction of quantification of QoS attributes a framework to model reputation based on user feedback is proposed. This approach uses past behavior of service providers and uses this knowledge to calculate reputation of a service. One thing that is worth considering here is that there could be a malicious user who could use a biased feedback to alter the reputation of the service, thus there is a need to include automated server side calculated attributes availability and reliability to give a precise estimate of reputation of service [1]. In [16] Feedback Forecasting Model is proposed that considers two major aspects during the rating process: First, to provide an automated feedback for customers who are fearful in giving feedback or do not bother to provide feedback. Second, to check what the feedback of a particular customer's credibility is. Here, user rating is considered for computing reputation and implicit QoS attributes are not considered. In [28] authors have used both availability and reliability to compute the reputation of the service. However, both attributes are used separately and their combined effect is not studied. Since availability gives the probability of success and reliability gives the change is failure trend, both must be used collectively to compute the reputation of a service. In [29] authors have proposed probabilistic methods to quantify QoS attributes: Cost, Throughput and Time, and studied their aggregated effect on composite service. However, the quantification of QoS attributes Availability, Reliability and their collective effect to compute reputation are not studied.

In the direction of the use of QoS at selection and composition following works are considered. In [17] the Ontology Web Language for Service (OWL-S) is used to perform a functional match among the available services and then these services are rated according to their QoS scores, however, the methodology of calculating the QoS score is not mentioned. A Relaxable QoS-based Service Selection (RQSS) algorithm is proposed in [19] that created a composite service by using heuristic techniques like MMKP (Multi-Dimensional Multi Choice Knapsack). The work studies the composition process for a composite service based on QoS attributes: Execution Time, Reliability, Availability, Reputation and Price. Here, reputation is based on user feedback. Also the combined behavior of availability and reliability to calculate reputation is not considered.

The novelties of this research are first, a probabilistic model for quantifying two different QoS attributes and second, modeling the reputation of a service.

\section{MODELING AND COMPOSITE QOS PARAMETERS TO APPROPRIATE WEB SERVICE SELECTION}

To quantify the major factors of QoS availability and reliability of atomic services using Markov Chain model and Weibull analysis respectively and reputation is modeled as an aggregation availability and reliability.

\section{Availability modeling}

Markov Chain model is used to study systems that could be represented as discrete states. 
Since service availability can have boolean discrete states we used Markov chain to model availability [1].

Service Availability is the probability that a service will be up and running. The main events that reflect status of service availability are the transition of a service from up to down state or down to up state. As an up/running service could fail due to any of these reasons like: server going down, hardware failure, internal logical error etc., these failure conditions are considered to a single down state [1].

Figure 1 depicts the state transitions for a service along with their transition probabilities. Value represents the transition probability of moving from state $i$ to state $j$ [1].

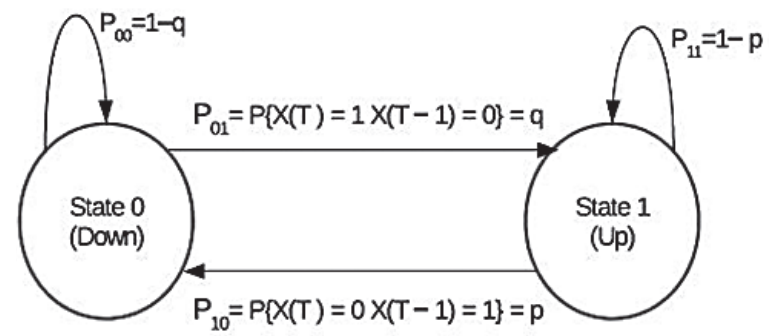

Fig. 1. Markov model for availability [1]

Where $p$ value is the transition probability from state up to state down, means a service is failed because of told reasons and not available, and $q$ value is the transition probability from state down to state up, means the fail service has been repaired and made available. All the transition probabilities could be represented in a form of Transition Probability Matrix $(P)$ as follows [1]:

$$
P=\left[\begin{array}{ll}
p_{00} & p_{01} \\
p_{10} & p_{11}
\end{array}\right]=\left[\begin{array}{cc}
(1-q) & q \\
p & (1-p)
\end{array}\right]
$$

Entries of Matrix $P\left(P_{i j}\right)$ correspond to the Markov Chain's single-path length transition probabilities. The row elements of matrix $P$ correspond to states that the system currently in and column elements denote the next state. Current transition matrix gives the transition probability of a single-path length. For better estimate of transition probabilities paths of all the possible lengths are considered to obtain $P^{n}$ matrix [1].

$$
\begin{aligned}
P^{n}= & {\left[\begin{array}{cc}
1-q & q \\
p & 1-p
\end{array}\right]^{n}=\frac{1}{p+q}\left[\begin{array}{ll}
p & q \\
p & q
\end{array}\right]+} \\
& +\frac{(1-p-q)^{n}}{p+q}\left[\begin{array}{cc}
q & -q \\
-p & p
\end{array}\right]
\end{aligned}
$$

For sufficiently large values of ' $n$ ' the results are interpreted as long run averages or limiting probabilities ' $P_{i}$ ' of system being in state ' $i$ '. In such cases $P^{n}$ reduces to [1]:

$$
\lim _{n \rightarrow \infty} P^{n}=\left[\begin{array}{cc}
\frac{p}{p+q} & \frac{q}{p+q} \\
\frac{p}{p+q} & \frac{q}{p+q}
\end{array}\right]
$$

From above it is concluded that availability is the probability of moving from state 1 to again state 1 in a path-length $n$. Thus the availability of the system on the long run can be said as [1]:

$$
\text { Availability }=P_{11}^{n}=\frac{q}{p+q}
$$

\section{Reliability modeling}

Reliability of a service is the rate of change of a service failure under test. Various probabilistic distribution mechanisms are used to study reliability. In a realistic scenario services' failure rate can be increasing, decreasing, or constant. To accommodate all these cases Weibull analysis has been considered. The following assumptions are considered to model reliability: $x=$ threshold for successful samples, $n=$ sample space in a given time frame, $F(x)=$ failure rate and $R(x)=$ reliability [1].

Failure of a service is a rare event as it does not occur as frequently as the success event, thus it is Poisson distributed. In the following failure of an atomic service is modeled. Failure rate $F(x)$ and reliability $R(x)$ can be related by [1]:

$$
F(x)=1-R(x)
$$

where: $\quad R(x)=e^{-\left[\frac{x}{\alpha}\right]^{\beta}}$

On simplification equation (5) can be written as:

$$
\ln \left[\ln \left(\frac{1}{1-F(x)}\right)\right]=\beta(\ln x)-\beta(\ln \alpha)
$$

Comparing parameters in Eq. (6) with that of straight line provides information that used to perform linear regression and this will provide the estimate for $\alpha$ and $\beta$ for computing the reliability of an atomic service. $\alpha$ parameter is the Weibull Characteristic Life and is a measure of spread in the distribution and $\beta$ is the Shape $\mathrm{Pa}-$ rameter that determine the nature of failure rate. In general failure rate of an atomic service $S_{i}$ is represented as [1]:

FailureRate $\left(S_{i}\right)=\left\{\begin{array}{c}\text { increasing, if } \beta>1.0 \\ \text { constant, if } \beta=1.0 \\ \text { decreasing, if } \beta<1.0\end{array}\right.$ 


\section{Reputation modeling}

Reputation is modeled as a composite QoS attribute comprising both availability and reliability.

Reputation $\left(S_{i}\right)=\min \left(\operatorname{Availability}\left(S_{i}\right)\right.$, Reliability $\left.\left(S_{i}\right)\right)$

To composite QoS parameters another method is used, as multiplying availability and reliability parameters. But the reason of using new method of minimum operation is that the availability and reliability values are in range $[0,1]$ and product of two numbers that smaller than 1 , will be very small, so the computed reputation value will be less than the value of minimum parameters means availability or reliability. Thus, it may be an appropriate service but not selected because of low reputation. But by computing reputation by using minimum operation, the obtained reputation value will not be lower than the minimum value of availability and reliability parameters and we have services with more reputation values. So services with more appropriate reputation will be selected.

\section{SIMULATION}

CPNTools is used for the simulation. This section is divided into two sub-sections:

1) input data,

2) simulation details.

\section{Input data}

In order to compute the QoS parameters, availability, reliability and reputation need to be counted. Failure count signifies the number of samples for which the service is found to be down and not responding. As the event of service going down is a rare event, thus a Poisson distribution is taken to model failure count. In this simulation is considered the acceptable failure count for an atomic service to be $10 \%$ of sample space. Such pattern of failure data is Poisson distributed with mean $(\mu)$ $=10 \%$ of sample space, such that the distribution generates $75 \%$ values in interval of $\mu \pm \delta$. Usage cost, another input data, signifies the cost that a user pays for a service. The usage cost of functionally same services generally lies in a range and thus uniformly distributed over that range.

\section{Simulation details}

The nets used in this simulation are depicted in Figures 2 - 4. Figure 2 represents the overall architecture of this approach in form of a hierarchical CPN. All the samples of all the services are taken as input for calculating availability and reliability. On the basis of Eq. (8) the net computes reputation of the selected service which lies in range $[0,1]$ with the name of Reputation 2 . Also this net computes the reputation of a service based on another type of reputation composite formula that is used for multiplication of parameters with the name of Reputation1 that continues to compare these results.

Figure 3 illustrates the process of computing availability. It takes all the samples of all the services as input and computes availability of one atomic service at a time. Availability for each atomic service is computed by dividing the sum of failure count with the total number of samples. One result token is generated for every atomic service reflecting its availability in the range $[0,1]$. A value in this range represents the probability of a service being up/running.

Figure 4 illustrates reliability of computation process. Samples of a selected service forms the input of the subnet. Linear regression is performed on these samples to evaluate regression parameters $S_{x}, S_{x x}, S_{y}, S_{v y}, S_{x y}$. Applying Weibull analysis on regression parameters reliability is computed in the range $[0,1]$. Here, the numeric value of reliability signifies the success trend, i.e. higher reliability value is higher success rate. the result also evaluates the Weibull shape parameter

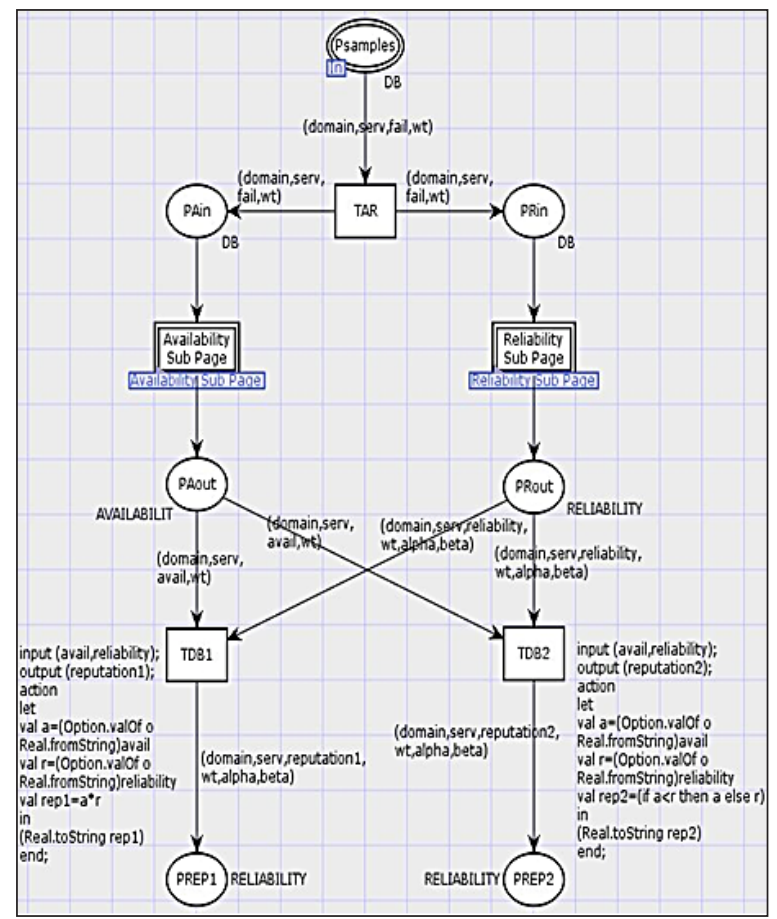

Fig. 2. Reputation modeling 
$\beta$ that represents failure trend. Figure 5 illustrates various colorsets used in creating the CPN nets for availability, reliability and reputation.

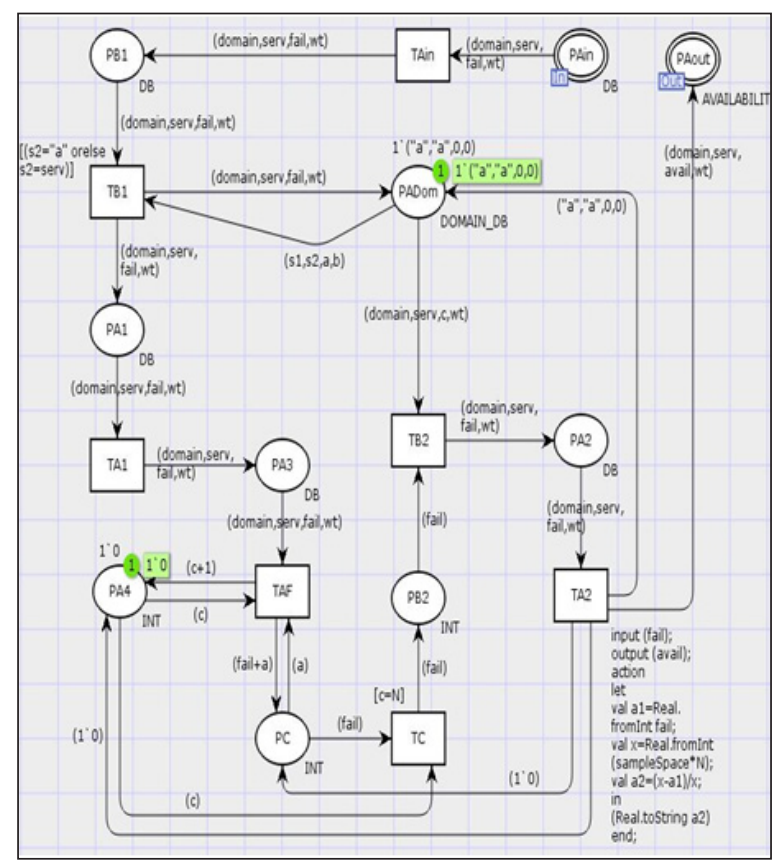

Fig. 3. Availability modeling

\section{EVALUATION RESULTS}

A series of experiments is performed to evaluate effectiveness, performance and feasibility of the proposed system. To study the QoS parameters, 20 functionally equivalent services are chosen for our experiment. The usage cost of these services is uniformly distributed in range [10,40]. The failure count of each service is Poisson distributed with of sample space. QoS parameters of services are studied for these samples.

Figure 6 illustrates modeling reputation based composite QoS. In this figure the reputation value is compared based on computation in several ways: by using availability and reliability parameters individually, by using the first composite formula [1] means multiplication of parameters, and also by using the second composite formula means minimum of parameters. If reputation is taken only on availability then service has highest reputation, and if based only on reliability then service has highest reputation. So, when these parameters are taken individually different estimates of reputation are generated. If reputation computes based on the first composite formula,

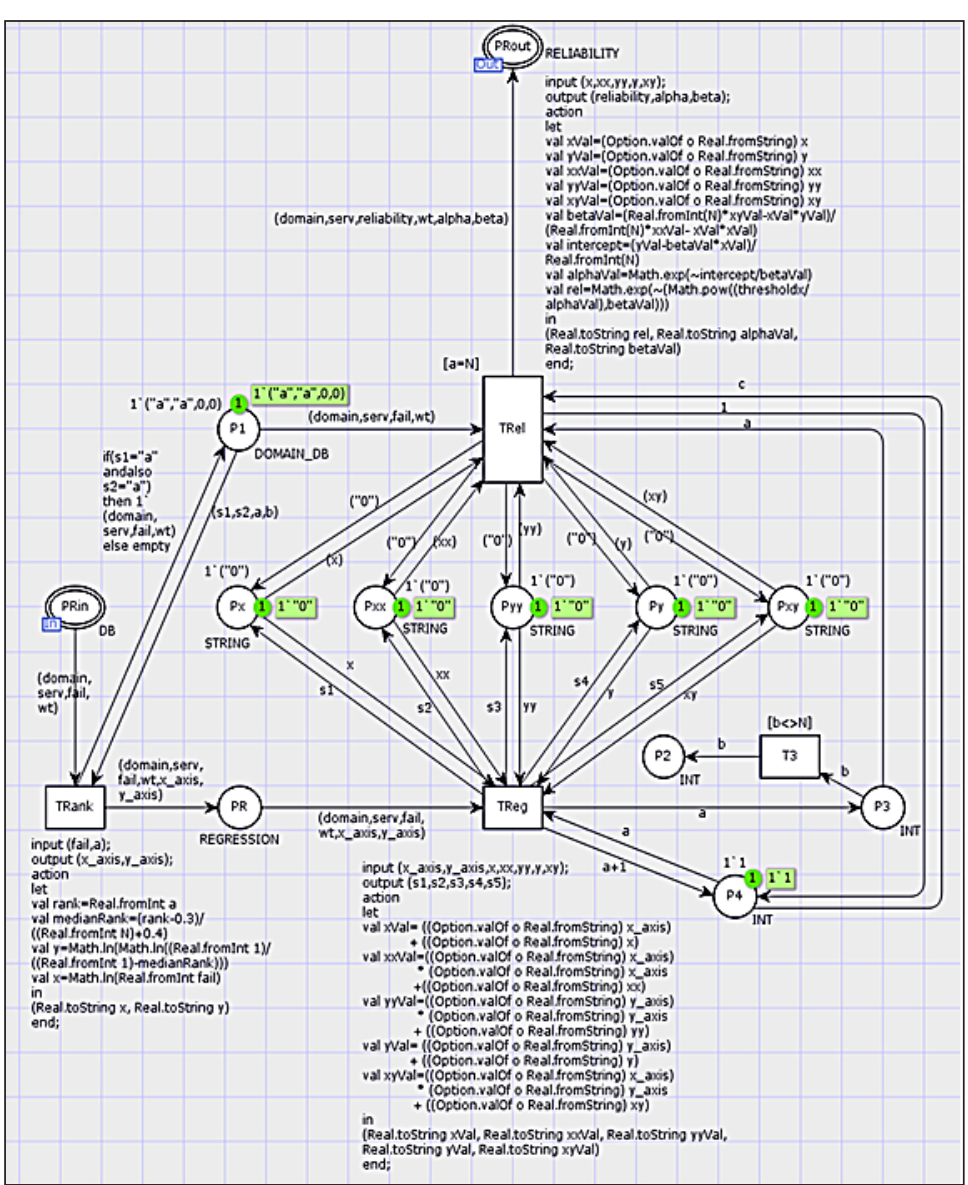

Fig. 4. Reliability modeling

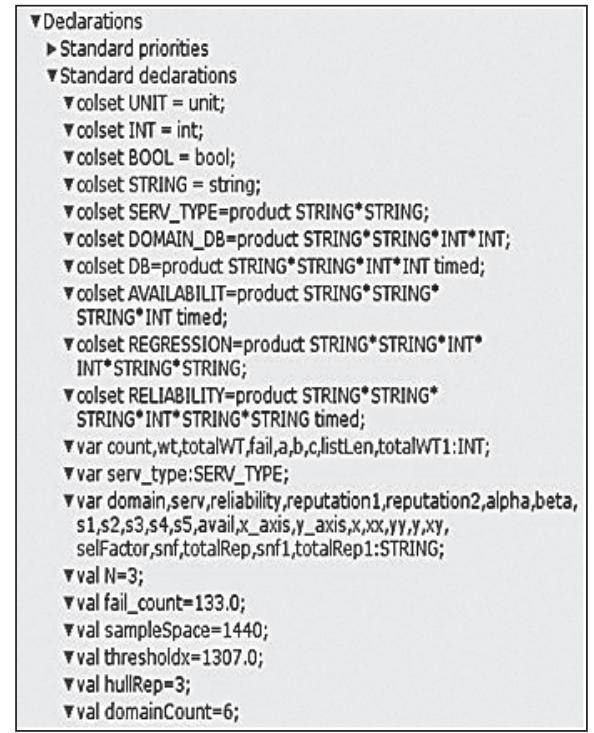

Fig. 5. CPN declarations 


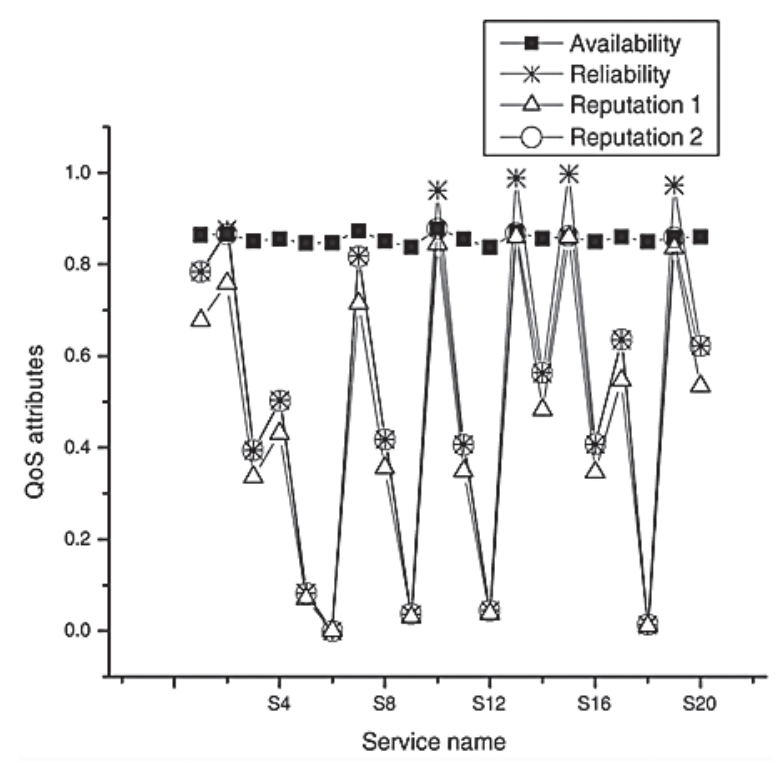

Fig. 6. Reputation based composite QoS

service has highest reputation, what that illustrates the combined effect of availability (probability of service being up/running) and reliability (failure trend of service), and gives a precise estimate of service reputation.

Reputation computing based on the second composite formula reputation value is improved in comparison to the first one. Because by computing the reputation based on the first composite formula the availability and reliability values are in the range $[0,1]$ and product of two numbers that smaller than 1, will be very small, so the computed reputation value will be less than the value of minimum parameters means availability or reliability. Thus it may be an appropriate service but not selected because of low reputation. But by computing reputation by using second composite formula, obtained reputation value will not be less than the minimum value of availability and reliability parameters and we have services with more reputation values. So services with more appropriate reputation will be selected. In this case service will have highest reputation.

Table 1 illustrates the values of availability, reliability, first type of reputation and second type of reputation. By comparing the values of first and second type of reputation, we can realize that the reputation is improved substantially.

Figure 7 illustrates the suitability of this proposed system from business perspective means usage cost than reputation. Generally a high reputation is considered to have high usage cost. But by using this approach there are services with high reputation and lower usage cost. Such a
Table 1. Availability, reliability and reputation values

\begin{tabular}{|l|c|c|c|}
\hline & Cost & Reputation1 & Reputation2 \\
\hline S1 & 11 & 0.6802 & 0.7858 \\
\hline S2 & 15 & 0.7627 & 0.8681 \\
\hline S3 & 17 & 0.3371 & 0.3953 \\
\hline S4 & 34 & 0.4326 & 0.5046 \\
\hline S5 & 22 & 0.0699 & 0.0824 \\
\hline S6 & 12 & 0.0000 & 0.0000 \\
\hline S7 & 32 & 0.7174 & 0.8199 \\
\hline S8 & 37 & 0.3570 & 0.4187 \\
\hline S9 & 36 & 0.0306 & 0.0365 \\
\hline S10 & 31 & 0.8475 & 0.8798 \\
\hline S11 & 14 & 0.3500 & 0.4082 \\
\hline S12 & 13 & 0.0375 & 0.0447 \\
\hline S13 & 28 & 0.8644 & 0.8728 \\
\hline S14 & 19 & 0.4848 & 0.5646 \\
\hline S15 & 26 & 0.8608 & 0.8610 \\
\hline S16 & 21 & 0.3475 & 0.4081 \\
\hline S17 & 20 & 0.5486 & 0.6363 \\
\hline S18 & 33 & 0.0109 & 0.0129 \\
\hline S19 & 24 & 0.8395 & 0.8610 \\
\hline S20 & 30 & 0.5374 & 0.6234 \\
\hline
\end{tabular}

service with highest reputation has a relatively lower usage cost ' 31 '.

Table 2 illustrates usage cost of each service and its reputation based on first and second type of formula. Thus, with prior usage cost we have services with higher reputation.

\section{CONCLUSION}

Web service reputation is an important parameter in QoS based web service selection. Selecting atomic services with high reputation helps creating robust, high performance, and cost ef-

Table 2. Usage cost and Reputation values

\begin{tabular}{|l|c|c|c|}
\hline & Cost & Reputation1 & Reputation2 \\
\hline S1 & 11 & 0.6802 & 0.7858 \\
\hline S2 & 15 & 0.7627 & 0.8681 \\
\hline S3 & 17 & 0.3371 & 0.3953 \\
\hline S4 & 34 & 0.4326 & 0.5046 \\
\hline S5 & 22 & 0.0699 & 0.0824 \\
\hline S6 & 12 & 0.0000 & 0.0000 \\
\hline S7 & 32 & 0.7174 & 0.8199 \\
\hline S8 & 37 & 0.3570 & 0.4187 \\
\hline S9 & 36 & 0.0306 & 0.0365 \\
\hline S10 & 31 & 0.8475 & 0.8798 \\
\hline S11 & 14 & 0.3500 & 0.4082 \\
\hline S12 & 13 & 0.0375 & 0.0447 \\
\hline S13 & 28 & 0.8644 & 0.8728 \\
\hline S14 & 19 & 0.4848 & 0.5646 \\
\hline S15 & 26 & 0.8608 & 0.8610 \\
\hline S16 & 21 & 0.3475 & 0.4081 \\
\hline S17 & 20 & 0.5486 & 0.6363 \\
\hline S18 & 33 & 0.0109 & 0.0129 \\
\hline S19 & 24 & 0.8395 & 0.8610 \\
\hline S20 & 30 & 0.5374 & 0.6234 \\
\hline
\end{tabular}




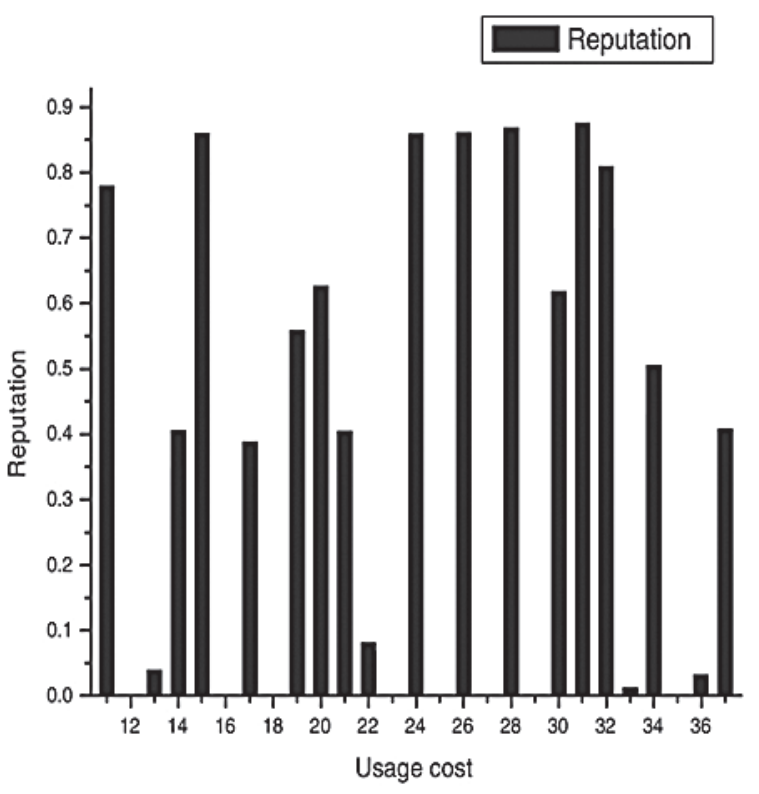

Fig. 7. Usage cost versus reputation

fective composite services. Availability focuses on probability of a service being up/running and reliability computes the failure trend of service. An appropriate composition of both factors, additionally giving a precise estimate of reputation, is resulted in selection of a service with high reputation and lower usage cost. Using these factors individually or inappropriate composition generates high reputation and high usage cost.

\section{REFERENCES}

1. Singh R.P., Pattanaik K.K. An approach to composite QoS parameter based web service selection. The 4th International Conference on Ambient Systems, Networks and Technologies ANT 2013. Procedia Computer Science 19, 2013, 470-477.

2. Rajeswari M., Sambasivam G., Balaji N., Basha M.S.S., Vengattaraman T., Dhavachelvan P. Appraisal and analysis on various web service composition approaches based on QoS factors. Journal of King Saud University - Computer and Information Sciences 26, 2014, 143-152.

3. Rostami M., Mohamadi M., Yaghoubi F., Nikrouz M. A proposed model for the standardization of web services discovery results using static and dynamic parameters. First National Conference on Electrical and Computer South of Iran, Khurmuj, 2013.

4. Mohammadi N., Mohsenzadeh M., Dezful M.A. Service discovery based on QoS parameters and service reputation. 3rd Iranian Conference on Electrical and Electronics Engineering, ICEEE 2011.

5. Parsa S., Aqdasinia H. An extensible quantitative model for the dynamic web services discovery. $16^{\text {th }}$
Iranian Conference on Electrical and Electronics Engineering, ICEEE 2008.

6. Khoshtarash A., Kheirkhah I., Jahan M.V. Evaluation and comparison of web service discovery and selection methods by syntactic and semantic procedures. $1^{\text {st }}$ National Conference on Applications of Intelligent Systems (Soft Computing) in Science and Technology, Islamic Azad Univercity of Quchan Branch, 4-5 March, 2013.

7. Rostami M., Malazizi L., Dejdar S. Provide a protocol to ensure reliable messaging between web services. $2^{\text {nd }}$ Conference on Computer, IT, Electrical and Electronic Engineering 2012.

8. Khoshtarash A., Mahmoudzadeh M., Abedini T., Mohammadzadeh J., Rahmanian S. An efficient ranking model based on qos for web service selection with consideration of user requirements. $1^{\text {st }}$ National Conference on Applications of Intelligent Systems (Soft Computing) in Science and Technology, Islamic Azad Univercity of Quchan Branch, 4-5 March, 2013.

9. Nayerifard T., Jobbehdari S., Modiri N. Web services security, review the latest guidelines. The First National Conference on New Approaches in Computer Engineering and Information Retrieval, Iran, 2013.

10. Khoshtarash A., Kheirkhah I., Jahan M.V. Evaluation of ranking methods for discovery and selection of optimum web service. $1^{\text {st }}$ National Conference on Applications of Intelligent Systems (Soft Computing) in Science and Technology, Islamic Azad Univercity of Quchan Branch, 4-5, March, 2013.

11. Banks J., Carson J., Discrete event simulation systems. Sharif University.

12. Law A.M., Kelton W.D. Simulation modeling and analysis. McGraw Hill, 2000.

13. Abernethy R.B. The weibull analysis handbook. $5^{\text {nd }}$ ed., Robert B. Abernethy, Florida 2006.

14. Scholz F. Weibull reliability analysis. FWS 5, 2002, 21-23.

15. Coles S. An introduction to statistical modeling of extreme values. Springer Verlag, 2001.

16. Al-Sharawneh J., Williams M.A., Goldbaum D. Web service reputation prediction based on customer feedback forecasting model. $14^{\text {th }}$ IEEE International Enterprise Distributed Object Computing Conference Workshops 2010, 33-40.

17. Benaboud R., Maamri R., Sahnoun Z.. Towards scalability of reputation and qos based web services discovery using agents and ontologies. Proceedings of the $13^{\text {th }}$ International Conference on Information Integration and Web-based Applications and Services, ACM, 2011, 262-269.

18. Martin D., Burstein M., Hobbs J., Lassila O., McDermott D., McIlraithm S. OWL-S: semantic markup for web services. Aug 6, 2011. 
19. Chia-Feng Lin, Ruey-Kai Sheu, Yue-Shan Chang, Shyan-Ming Yuan. A relaxable service selection algorithm for QoS-based web service composition. Information and Software Technology 53(12), 2011, 1370-1381.

20. Esfahani P.M., Broumandnia A. Clustering web services based on the qualitative features. The First National Conference on New Approaches in Computer Engineering and Information Retrieval, Iran, 2013.

21. Giallonardo E., Zimeo E. More semantics in QoS matching. Proceedings of the IEEE International Conference on Service-Oriented Computing and Applications, Newport Beach, CA, 2007, 163-171.

22. Sha L., A QoS based Web service selection model. Proceedings of the International Forum on Information Technology and Applications, Chengdu, 2009, 353-356.

23. Yan J., Piao J., Towards QoS-based web service discovery. Proceedings of the International Conference on Service Oriented Computing, Sydney, 2009, 200-210.

24. Chifu V.R., Salomie I., Dinsoreanu M., David T., Acretoaie V. Ant-inspired technique for automatic web service composition and selection. $12^{\text {th }}$ Inter- national Symposium on Symbolic and Numeric Algorithms for Scientific Computing, 2010.

25. Kossmann D., Borzsony S., Stocker K. The skyline operator. Proceedings of the $17^{\text {th }}$ International Conference on Data Engineering, Heidelberg, 2001, 421-430.

26. Skoutas D., Sacharidis D., Simitsis A., Sellis T. Ranking and clustering web services using multicriteria dominance relationships. IEEE Transactions on Services Computing, 3(3), 2012, 163-177.

27. Mahdavi F., Motameni H., Momeni H. Automatic generation of web services based on genetic-fuzzy approach. First National Innovation Conference on Computer Engineering and Information Technology, Tonekabon, Iran, 2013.

28. Janardhan D.B., Devane S.R.. Web service reputation-based search agent. IEEE Student Conference on Research and Development (SCOReD) 2009, 184-187.

29. Zheng H., Yang J., Zhao W. Probability distribution-based QoS analysis for web service composition. [In:] Dickson Chiu et al. (eds.) Web Information Systems Engineering WISE 2010 Workshops, Lecture Notes in Computer Science, 6724, 98111, 2011. 